\title{
A NEW PROOF OF THE EXISTENCE OF A TRACE IN A FINITE VON NEUMANN ALGEBRA
}

\author{
BY F. J. YEADON
}

Communicated by Peter D. Lax, April 28, 1970

The construction of the dimension function for projections in the various types of factor, and the definition of the trace in a factor of type $\mathrm{II}_{\mathbf{1}}$ first appeared in a classical paper of Murray and von Neumann [7]. The proof of the additivity and weak continuity of the trace appeared in [8]. Subsequent authors [2], [5], [6] have demonstrated the existence of traces on a larger class of von Neumann algebras, but all have employed some variant of the Murray-von Neumann method of proof. The purpose of the present paper is to provide a short and independent proof of the following theorem.

TheOREM. Let $\mathbb{R}$ be a finite von Neumann algebra, with centre $\mathfrak{C}$, and let $\mathcal{U}$ be the group of unitary elements of $R$.

(1) If $h$ is an ultraweakly continuous linear form on $\mathfrak{C}$, then there is a unique linear form $g$ on $R$ such that

(i) $g$ is ultraweakly continuous,

(ii) $g(A)=g\left(U^{*} A U\right)$ for $A \in R$ and $U \in \mathcal{U}$,

(iii) $g(C)=h(C)$ for $C \in \mathbb{C}$.

Moreover $\|g\|=\|h\|$, and if $h$ is positive then $g$ is positive.

(2) There is a unique linear mapping $T: \Omega \rightarrow \mathcal{C}$ such that

(i) $T$ is ultraweakly continuous,

(ii) $T$ is positive, and $T(I)=I$,

(iii) $T\left(U^{*} A U\right)=T(A)$ for $A \in \Omega$ and $U \in \mathcal{U}$,

(iv) $T(C A)=C \cdot T(A)$ for $A \in \mathbb{R}$ and $C \in \mathcal{C}$.

The terminology is that of [3], except that finite is used here in the sense that if $E$ is any projection in $Q$ that is equivalent to $I$ then $E$ $=I$. A positive linear form $g$ on $R$ satisfying (i) and (ii) of (1) is called a finite normal trace on $R$. The mapping $T$ in part (2) is the canonical centre-valued trace of $R$.

The "uniqueness" part of (1) and the deduction of (2) from (1) are straightforward. The "existence" part of (1) will be proved by the application of a fixed point theorem. We first require two lemmas.

AMS 1970 subject classifications. Primary 46L10; Secondary 46L10, 46L25.

Key words and phrases. Finite von Neumann algebra, finite normal trace, canonical centre-valued trace, comparison of projections, ultraweakly continuous linear form, Ryll-Nardzewski fixed point theorem. 
Lemma 1. Let $E$ and $F$ be projections in a finite von Neumann algebra $R$ and let $\left(E_{k}\right)$ be a sequence of projections in $R$ such that $\sup E_{k}=E$, and $E_{k} \leqq E_{k+1}, E_{k} \prec F$ for all $k$. Then $E \prec F$.

Proof. Let $E_{1} \sim F_{1} \leqq F$. Let $k \geqq 1$ and suppose that projections $F_{1} \cdots F_{k} \in \Omega$ have been chosen so that $F_{i} F_{j}=0$ for $1 \leqq i<j \leqq k$, $\sum_{i=1}^{k} F_{i} \leqq F$, and $E_{i+1}-E_{i} \sim F_{i+1}$ for $1 \leqq i \leqq k-1$. Since $E_{k+1} \prec F$ and $Q$ is finite, $I-E_{k+1} \succ I-F$. Since also $E_{k} \sim \sum_{i=1}^{k} F_{i}$, it follows that

$I-\left(E_{k+1}-E_{k}\right) \succ I-\left(F-\sum_{i=1}^{k} F_{i}\right), \quad E_{k+1}-E_{k} \prec F-\sum_{i=1}^{k} F_{i}$,

and we can choose $F_{k+1}$ so that $E_{k+1}-E_{k} \sim F_{k+1} \leqq F-\sum_{i=1}^{k} F_{i}$. Thus there is a sequence of projections $\left(F_{k}\right)$ such that $F_{i} F_{j}=0$ for $i \neq j$, $\sum_{i=1}^{\infty} F_{i} \leqq F$, and $E_{k+1}-E_{k} \sim F_{k+1}$ for all $k$. Hence

$$
E=E_{1}+\sum_{i=1}^{\infty}\left(E_{i+1}-E_{i}\right) \sim \sum_{i=1}^{\infty} F_{i} \leqq F .
$$

Let $R_{*}$ denote the dual of $R$ for the ultraweak topology, and for each $U \in \mathcal{U}$, let $T_{U}$ be the linear isometry of $\Omega_{*}$ onto itself such that $\left(T_{U} f\right)(A)=f\left(U^{*} A U\right)$ for all $f \in \Re_{*}$ and $A \in R$.

Lemma 2. Let $R$ be a finite von Neumann algebra, let $f \in R_{*}$, and let $Q$ be the closed convex hull in $R_{*}$ of the set $K=\left\{T_{U} f: U \in \mathcal{U}\right\}$. Then $Q$ is weakly compact.

Proof. By $[4$, V.6.4 $]$ it is sufficient to show that $K$ is weakly relatively compact. If $K$ is not weakly relatively compact, then by [1, Theorem II.2(2)] there is a sequence $\left(E_{n}\right)$ of mutually orthogonal projections in $R$, a sequence $\left(f_{n}\right)$ in $K$, and a real positive $\epsilon$, such that $\left|f_{n}\left(E_{n}\right)\right| \geqq \epsilon$ for all $n$. Let $U_{n} \in \mathcal{U}$ be such that $f_{n}=T_{U_{n}} f$, and let $F_{n}=U_{n}^{*} E_{n} U_{n}$, so that $\left(F_{n}\right)$ is a sequence of projections in $R$ such that $F_{n} \sim E_{n}$ and $\left|f\left(F_{n}\right)\right|=\left|f\left(U_{n}^{*} E_{n} U_{n}\right)\right|=\left|\left(T_{U_{n}} f\right)\left(E_{n}\right)\right|=\left|f_{n}\left(E_{n}\right)\right| \geqq \epsilon$ for all $n$. Let $P_{n}=\sum_{m \text { zn }} E_{m}, Q_{n}=\sup _{m \geq n} F_{m}$, so that $P_{n+1} \leqq P_{n}, Q_{n+1} \leqq Q_{n}$ for all $n$, and let $G=\inf Q_{n}$. Let $n$ now be fixed and for each $k$ let $R_{k}=\sup \left\{F_{i}: n \leqq i \leqq n+k\right\}$. Suppose that $k \geqq 1$ and $R_{k-1} \prec \sum_{i=n}^{n+k-1} E_{i}$. Now

$$
R_{k}=R_{k-1}+\left(\sup \left\{R_{k-1}, F_{n+k}\right\}-R_{k-1}\right)
$$

and

$$
\sup \left\{R_{k-1}, F_{n+k}\right\}-R_{k-1} \sim F_{n+k}-\inf \left\{R_{k-1}, F_{n+k}\right\} \leqq F_{n+k} \sim E_{n+k},
$$

by [3, III.1.1, Corollary 1]. Hence $R_{k} \prec \sum_{i=n}^{n+k} E_{i}$, and it follows that $R_{k} \prec \sum_{i=n}^{n+k} E_{i} \leqq P_{n}$ for all $k$, and by Lemma 1 , that $Q_{n}=\sup R_{k} \prec P_{n}$. 
Since $R$ is finite, $I-P_{n} \prec I-Q_{n} \leqq I-G$ for all $n$, and again by Lemma $1, I=\sup \left(I-P_{n}\right) \prec I-G$, whence $G=0$. Hence $\left(F_{n}\right)$ converges ultraweakly to 0 , which contradicts $\left|f\left(F_{n}\right)\right| \geqq \epsilon>0$ for large $n$.

There is an obvious analogy between the above method of proof and the statement of [10, Theorem 8].

Proof of Theorem. (1) Let $f \in Q_{*}$ be chosen so that $f(C)=h(C)$ for $C \in \mathcal{C}$, let $Q$ be the set defined in Lemma 2, and let $s$ be the group $\left\{T_{U}: U \in \mathcal{U}\right\}$ acting on $Q$. The set $Q$ is weakly compact by Lemma 2 , and $\delta$ is obviously noncontracting in the sense of [11, Definition]. Hence, by the Ryll-Nardzewski fixed point theorem [11, Theorem 3 ], [9], where we take the locally convex space $E$ to be $\mathcal{R}_{*}$ with the norm topology, there is an element $g \in Q$ such that $T_{U} g=g$ for all $U \in \mathcal{U}$, that is, $g\left(U^{*} A U\right)=g(A)$ for all $A \in R$ and $U \in \mathcal{U}$. If $C \in \mathfrak{e}$, then, for all $U \in \mathcal{U}, U^{*} C U=C,\left(T_{U} f\right)(C)=f\left(U^{*} C U\right)=f(C)=h(C)$, $k(C)=h(C)$ for any $k \in Q$, hence $g(C)=h(C)$.

Now let $g$ be any linear form on $\Omega$ satisfying (i), (ii), (iii), and let $g=|g| \cdot V$ be the polar decomposition of $g$. Then for any $U \in \mathcal{U}$, $T_{U} g=\left(T_{U}|g|\right) \cdot\left(U V U^{*}\right)$ is the polar decomposition of $T_{U} g(=g)$. By uniqueness of the polar decomposition, $U V U^{*}=V$ for all $U \in \mathcal{U}$, so that $V \in \mathcal{C}$ and $\|g\|=g\left(V^{*}\right)=h\left(V^{*}\right) \leqq\|h\|$. Since obviously $\|g\| \geqq\|h\|$, we have $\|g\|=\|h\|$. An application of the preceding argument with $h=0$ suffices to prove uniqueness. If $h$ is positive, then $g(1)=h(1)$ $=\|h\|=\|g\|$, and so $g$ is positive.

(2) By part (1) we can define a linear isometry $T_{*}: \mathfrak{C}_{*} \rightarrow \mathfrak{Q}_{*}$ such that

(a) $\left(T_{*} h\right)\left(U^{*} A U\right)=\left(T_{*} h\right)(A)$,

(b) $\left(T_{* h}\right)(C)=h(C)$,

for $h \in \mathfrak{C}_{*}, A \in \Re, U \in \mathfrak{U}$ and $C \in \mathcal{C}$. Let $T: R \rightarrow \mathfrak{C}$ be the conjugate mapping. Since the ultraweak topology agrees with the weak* topology when $R$ is identified with the dual of $\Omega_{*}$, (i) is immediate. (ii) and (iii) are easily verified; (iv) and the uniqueness of $T$ follow from the uniqueness proved in (1).

\section{REFERENCES}

1. C. A. Akemann, The dual space of an operator algebra, Trans. Amer. Math. Soc. 126 (1967), 286-302. MR 34 \#6549.

2. J. Dixmier, Les anneaux d'opérateurs de classe finie, Ann. Sci. École. Norm. Sup. (3) 66 (1949), 209-261. MR 11, 370.

3. - Les algèbres d'operateurs dans l'espace Hilbertien, Paris, 1969.

4. N. Dunford and J. T. Schwarz, Linear operators. I: General theory, Interscience, New York, 1963. MR $32 \# 6181$.

5. R. V. Kadison, On the additivity of the trace in finite factors, Proc. Nat. Acad. Sci. U.S.A. 41 (1955), 385-387. MR 16, 1033. 
6. - The trace in finite operator algebras, Proc. Amer. Math. Soc. 12 (1961), 973-977. MR 25 \#3385.

7. F. J. Murray and J. von Neumann, On rings of operators, Ann. of Math. (2) 37 (1936), 116-229.

8. - On rings of operators. II, Trans. Amer. Math. Soc. 41 (1937), 208-248.

9. I. Namioka and E. Asplund, A geometric proof of Ryll-Nardzewski's fixed point theorem, Bull. Amer. Math. Soc. 73 (1967), 443-445. MR 35 \#799.

10. C. Ryll-Nardzewski, Generalized random ergodic theorems and weakly almost periodic functions, Bull. Acad. Polon. Sci. Sér. Sci. Math. Astronom. Phys. 10 (1962), 271-275. MR 30 \#225.

11. - On fixed points of semigroups of endomorphisms of linear spaces, Proc. Fifth Berkeley Sympos. Math. Statist. and Probability (Berkeley, Calif., 1965/66), vol. II : Contributions to Probability Theory, part I, Univ. of California Press, Berkeley, Calif., 1967, pp. 55-61. MR 35 \#5977.

University of Hull, Hull, England 